\title{
Bidirectional Control of Risk-Seeking Behavior by the Basolateral Amygdala
}

\author{
(D) J. Miguel Cisneros-Franco, ${ }^{1,2}$ and ${ }^{-D E t i e n n e ~ d e ~ V i l l e r s-S i d a n i ~}{ }^{1,2}$
}

DOI:http://dx.doi.org/10.1523/ENEURO.0168-18.2018

${ }^{1}$ Department of Neurology and Neurosurgery, Montreal Neurological Institute, McGill University, Montreal, QC H3A 2B4, Canada and ${ }^{2}$ Centre for Research on Brain, Language, and Music, Montreal, QC H3G 2A8, Canada

Key words: basolateral amygdale; decision-making; optogenetics; punishment; reward; risk

\section{Significance Statement}

Decision-making under risk entails the possibility of simultaneously receiving positive (reward) and negative (punishment) stimuli. To learn in this context, one must integrate conflicting information related to the magnitude of reward and the probability of punishment. Long-term inactivation of the basolateral amygdala (BLA) disrupts this process and increases risky behavior. In a recent study published in the Journal of Neuroscience, Orsini et al. (2017) showed that briefly inhibiting the BLA may result in increased or decreased risk-taking behavior, depending on the phase of the decision process in which BLA activity is disrupted. Here, we discuss the results and propose future experiments that could improve our understanding of how the BLA contributes to adaptive learning under risk and uncertainty.

In complex, "real-world" environments, choices made may result in both rewards and adverse outcomes, each associated with different and often-changing probabilities. Decision-making under risk and uncertainty requires sustained attention and constant updating of learned rules to adequately valuate available alternatives. During risky decision-making, rewarding or punishing outcomes encountered following each decision facilitate learning through positive or negative reinforcement, respectively (Wächter et al., 2009). How individuals react to such competing environmental cues has been the focus of numerous studies in decision neuroscience (Preuschoff et al., 2015). These studies show that cultural, social, and genetic factors shape risk preference, although transient internal states, such as mood, fatigue, or recent experience, may also influence an individual's propensity to risk-taking (Weber and Johnson, 2009).

Located at the crossroads of corticolimbic circuits that mediate reinforcement learning, the basolateral amygdala (BLA) responds to arousing stimuli of both positive and

Received April 30, 2018; accepted June 28, 2018; First published July 6, 2018. The authors declare no competing financial interests.

Author contributions: J.M.C.-F. and E.d.V.-S. wrote the paper.

This work was supported by the Canadian Institutes of Health Research.

Correspondence should be addressed to J. Miguel Cisneros-Franco, 3801 University Street, Room 753, Montreal, QC H3A2B4, Canada, E-mail: negative valence (Shabel and Janak, 2009) and is necessary for the establishment of reward associations (Baxter and Murray, 2002) and fear conditioning (Krabbe et al., 2017). This functional heterogeneity has made the BLA a prime target to study associative learning using risky decision-making tasks (RDTs), in which rodents have to choose between a small, "safe" food reward and a large, "risky" reward that might be paired with a punishment. Over the last decade, studies using RDTs have shown that pharmacological inactivation and lesions of the BLA increase risk-seeking behavior (Orsini et al., 2015; Piantadosi et al., 2017). However, studies with high temporal resolution allowing trial-by-trial manipulation of BLA activity are lacking. Therefore, it remains unknown whether increased risk-seeking behavior is the result of BLA lossof-function specifically during the deliberation phase, i.e., in decision-making per se, and/or during the outcome phase, i.e., the associative phase of reward and punishment.

mike.cisneros-franco@mail.mcgill.ca.

DOI:http://dx.doi.org/10.1523/ENEURO.0168-18.2018 Copyright (C) 2018 Cisneros-Franco and de Villers-Sidani

This is an open-access article distributed under the terms of the Creative Commons Attribution 4.0 International license, which permits unrestricted use, distribution and reproduction in any medium provided that the original work is properly attributed. 
A

\begin{tabular}{|l|l|}
\hline Deliberation Outcome & Intertrial interval \\
\hline
\end{tabular}

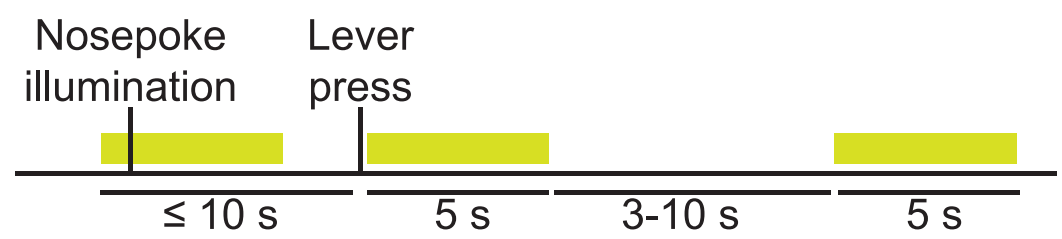

B Reward- and punishment-encoding cells in BLA

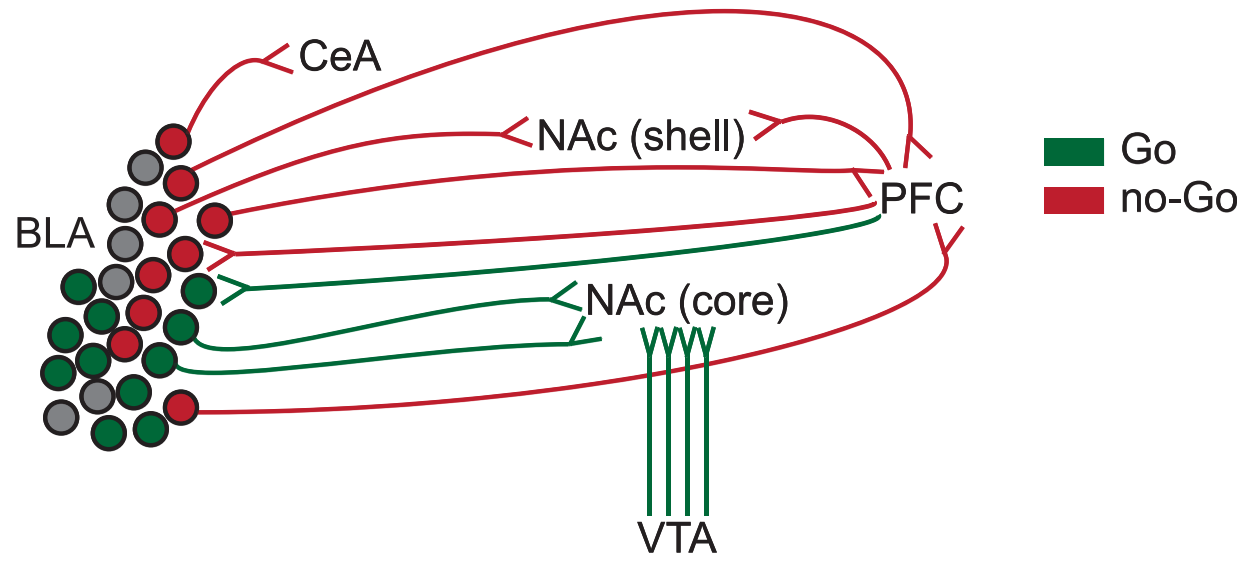

\section{BLA inhibition during punished reward}

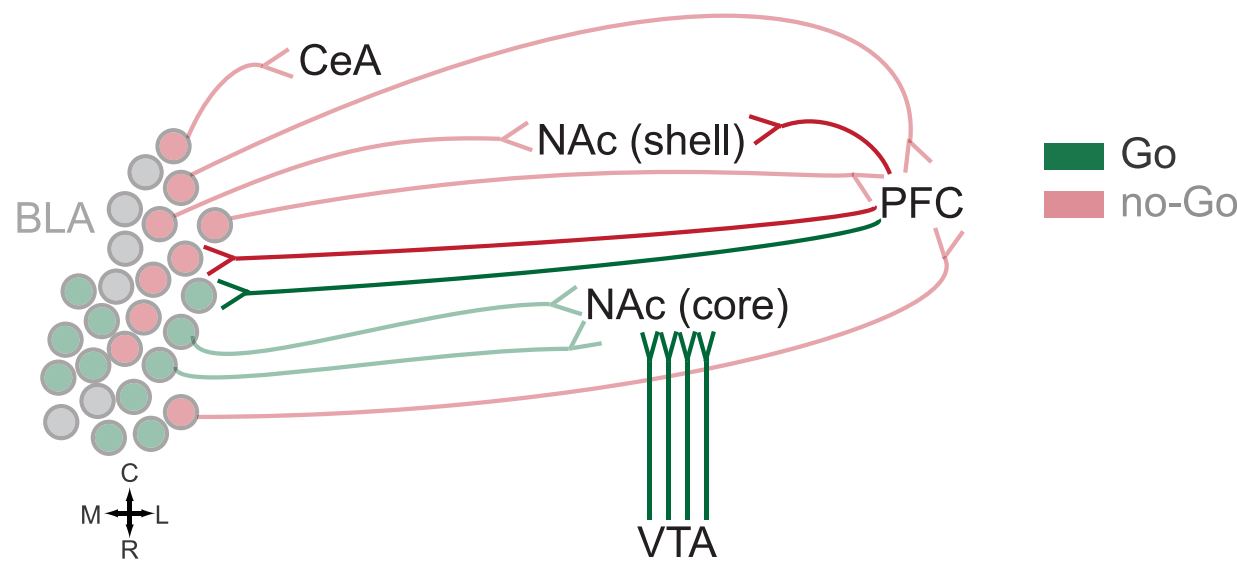

Figure 1. A, Experimental paradigm used in Orsini et al. (2017). The RDT was divided in three phases: deliberation, outcome, and intertrial interval. Optogenetic inhibition of the BLA occurred during one of the three phases and lasted $\leq 5 \mathrm{~s}$. B, Reward (green) and punishment (red) are processed by distinct subpopulations within the BLA. Only major afferent and efferent BLA connections discussed in the text are shown. $\boldsymbol{C}$, Concurrent delivery of a large reward alongside punishment results in conflicting information, of positive and negative valence, respectively, reaching the BLA. Hypothesis: in absence of no-Go inputs from BLA to CeA, NAc shell, and PFC, predominance of Go inputs to the NAc (notably from VTA) may bias the animal's perceived experience toward rewarding stimuli, thus increasing risky choice. $\mathrm{R}$, rostral; $\mathrm{C}$, caudal; $\mathrm{M}$, medial; $\mathrm{L}$, lateral.

In a study published in the Journal of Neuroscience, Orsini et al. (2017) addressed this gap in knowledge by using optogenetics to inhibit BLA activity at three different phases of a RDT; namely, deliberation, outcome, and intertrial interval. The deliberation phase corresponded to the $\leq 10$-s period during which rats had to choose between two levers (safe, associated with a small reward, or risky, associated with a large reward that was accompa- nied by an increasing probability of a footshock). Lever choice marked the beginning of the 5-s outcome period during which reward (always) and punishment (if any) were delivered, followed by a variable intertrial interval that brought each trial's length to $40 \mathrm{~s}$. Laser delivery occurred during one of the three phases and lasted $\leq 5 \mathrm{~s}$ (Fig. 1A).

In the control condition, as expected, the likelihood of choosing the risky reward diminished as the probability of 
punishment increased. Interestingly, transient BLA inactivation yielded opposite results depending on the phase of the decision-making process at which it occurred. On the one hand, BLA inhibition during the deliberation phase decreased risky choice and resulted in a higher probability of performing a lose-shift, i.e., making a safe choice immediately after punishment. On the other hand, silencing of BLA neurons during delivery of a large, punished outcome, increased risky choice, and decreased the proportion of lose-shift trials.

How to reconcile such disparate results? One possible explanation comes from the fact that optogenetic inhibition reduced overall BLA activity, without cell-type specificity. Because the BLA receives inputs from and sends projections to different cortical and subcortical targets (O'Neill et al., 2018), it is important to consider its functional and anatomic heterogeneity before discussing Orsini et al.'s results.

Inputs reaching the BLA from the prefrontal cortex (PFC) and dopaminergic neurons in the ventral tegmental (VTA) modulate reward sensitivity. Disruption of PFC-toBLA projections, pharmacological blockade of $D_{1}$ receptors $\left(D_{1} R\right)$, and $D_{2} R$ stimulation all reduce risky choice (St Onge et al., 2012; Larkin et al., 2016). $D_{1} R$ stimulation however may increase or decrease risky choice, depending on reward probabilities and individual risk-preferences (Larkin et al., 2016).

Regarding BLA outputs, recent evidence suggests that risk-seeking and risk-avoidance are mediated by distinct neuronal subpopulations within the BLA, which in turn project to segregated brain regions driving opposing outcomes (Namburi et al., 2015; Beyeler et al., 2016, 2018). BLA neurons that synapse in the nucleus accumbens (NAc projectors) are preferentially excited to rewardpredictive cues (Beyeler et al., 2016), whereas central amygdala $(\mathrm{CeA})$ projectors and medial PFC (mPFC) projectors are preferentially excited to cues associated with an aversive outcome (Burgos-Robles et al., 2017; Fig. 1B). In contrast, neurons projecting to the ventral hippocampus do not show a preference for either positive or negative stimuli (Beyeler et al., 2016). Moreover, a study by the same research group showed that specific activation of BLA NAc projectors and CeA projectors facilitate positive and negative reinforcement learning, respectively (Namburi et al., 2015). Taken together, these results suggest that distinct BLA subpopulations are engaged differently at discrete time-points of the decision-making process. Hence, it is possible that non-specific inhibition of pre- and/or postsynaptic activity within the amygdala might have selective effects depending on the phase of the decision-making process that is disrupted.

\section{BLA Inhibition during Delivery of Large, Punished Outcome Increases Risky Choice}

Delivery of a large reward alongside punishment results in conflicting positive- and negative-valence signals simultaneously reaching the BLA. The increase in riskseeking behavior reported by Orsini et al. (2017) with BLA inactivation during delivery of a large, punished reward suggests that intact BLA function contributes to the integration of reward magnitude and punishment-related information.

Given the role of BLA NAc-, CeA-, and mPFCprojecting neurons in reinforcement learning, it seems sensible to hypothesize that conflicting outcomes are represented in the BLA by opposing yet complementary activity of these neuronal populations. The increase of risky choice and the reduction in the number of lose-shift trials observed by Orsini et al. (2017) suggests that, in the presence of conflicting inputs, activity of $\mathrm{CeA}$ and/or mPFC projectors is more determinant than that of NAc projectors in informing subsequent decisions. If, indeed, discrete BLA subpopulations are preferentially recruited during the outcome phase, inhibition of all projection neurons would have an impact only on the neurons that are normally activated during said phase. This raises the question of why, in the absence of BLA to NAc inputs, risk-seeking behavior is not affected. According to our current understanding of NAc reward circuitry, there are at least two possible, non-mutually exclusive, explanations for this result. The first possible explanation relates to the functional and anatomic subdivisions of the NAc, while the second has to do with other brain structures besides the BLA that feed into the NAc.

First, the BLA projects to both the lateral ("core") and medial ("shell") subdivisions of the NAc. A recent study showed that pharmacological inactivation of either BLA or NAc shell during a RDT increased risky behavior, suggesting that both structures suppress punished reward seeking (Piantadosi et al., 2017). In contrast, NAc core inactivation reduced overall responding, even in the absence of any risk, suggesting that NAc core facilitates reward-seeking, independent of motivational conflict (Piantadosi et al., 2017). It is therefore possible that nonspecific BLA inactivation affected a BLA-NAc shell circuit responsible for the punishment-induced inhibition of behavior.

Second, the NAc is a major target of dopaminergic VTA neurons, which encode the value of predicted and observed rewards and respond strongly to rewards during the course of learning (Hollerman and Schultz, 1998). Moreover, VTA stimulation following a "risky loss," i.e., punishment in the absence of reward, increases risk preference (Stopper et al., 2014). Taken together, these observations indicate that BLA silencing during delivery of a large, punished reward might result in or resemble the effect of reduced NAc shell activity, effectively "releasing" the NAc core, which may also respond to strong VTA inputs that bias the animal's perceived experience toward rewarding stimuli (Fig. 1C).

\section{BLA Inhibition during Deliberation Decreases Risky Choice}

The result of decreased risky choice with BLA inactivation during deliberation observed by Orsini et al. (2017) appears to be at odds with previous reports using lesions and pharmacologic inhibition of the BLA (Orsini et al., 2015; Piantadosi et al., 2017). Nevertheless, as mentioned above, said techniques do not offer the time resolution 
needed to study the role of the BLA during different phases of the RDT, a limitation that was circumvented by the use of optogenetics. Therefore, the experiments conducted by Orsini et al. (2017) demonstrate that previously observed deficits in decision-making following BLA inactivation are the result of BLA loss-of-function that specifically affected the integration of conflicting outcomes and not the deliberative process itself. Given that decreased risk-seeking behavior was elicited exclusively with BLA silencing during the deliberation phase, it remains to be seen which network(s) within the BLA inform decisionmaking during such a brief period, lasting no more than 5 $s$ in the study by Orsini et al. (2017). A viable approach to answer this question would be the use of optogenetics to identify ("phototag") BLA subpopulations by simultaneously injecting a Cre-dependent opsin construct in the $B L A$ and a construct carrying Cre recombinase in the structure where the population of interest projects (Beyeler et al., 2018). Should a neuronal BLA subpopulation be identified as key in decision-making during deliberation, this approach could be used in combination with computational models developed for functional neuroimaging to better characterize any observed activity patterns (Prévost et al., 2013). Of particular interest would be to test whether the BLA performs model-based computations, in which the value of actions are updated using a rich representation of the structure of the decision problem, as opposed to purely prediction-error driven model-free algorithms (Corrado and Doya, 2007).

\section{Concluding Remarks and Future Directions}

The main contribution of the study by Orsini et al. (2017) is the demonstration that the BLA plays different roles at different behavioral phases of risky decision-making. While these findings may have implications for the study of impulse control disorders, it should be noted that certain aspects of risky decision-making can be encountered in everyday situations and may have real-life consequences with respect to personal finances. For instance, Knutson et al. (2011) showed that individuals who were better at positive reinforcement learning had more assets, whereas those who were more effective at learning from negative outcomes had less debt. In a subsequent study, the authors found that reduced impulse control, but not cognitive abilities, was the main factor that predicted an individual's susceptibility to investment fraud (Knutson and Samanez-Larkin, 2014).

Future studies may build on the work of Orsini et al. (2017) to further our understanding of how the BLA contributes to adaptive learning in the context of risk and uncertainty. To this end, we propose two avenues of research going forward. First, investigate if the effects of BLA inhibition vary as a function of individual differences in risk propensity. Although most subjects show a marked bias toward risk aversion, a few seem to prefer risk. Recent studies have shown that risk-seeking rats could be "converted" to risk-averse rats using phasic optogenetic stimulation of $D_{2} R$ neurons in the NAc (Zalocusky et al., 2016) or $D_{2} R$ agonists infused into the BLA (Larkin et al., 2016). Thus, a priori identification of risk-prone and risk-averse subjects may reveal whether acute BLA inhibition has the same effect on behavior regardless of previously established risk preference.

Second, it remains to be seen whether repeated transient manipulation of BLA activity over an extended period of time will result in significant and long-lasting changes in reward processing. For instance, chronic mPFC stimulation in rats reduces reward-seeking behavior and gives rise to brain-wide activity patterns that predict the onset and severity of anhedonia (Ferenczi et al., 2016). Given the numerous reciprocal connections between the BLA and other brain regions, including the mPFC, we posit that chronic manipulation of BLA activity could similarly change corticolimbic synchrony and alter reward sensitivity. Experiments using intermittent optogenetic or sustained chemogenetic manipulation of BLA activity could be used to test this hypothesis. Going forward, research with a focus on brain networks, rather than on specific isolated structures, may best reveal the mechanisms whereby different behaviors arise from common brain regions.

\section{References}

Baxter MG, Murray EA (2002) The amygdala and reward. Nat Rev Neurosci 3:563-573. CrossRef

Beyeler A, Namburi P, Glober GF, Simonnet C, Calhoon GG, Conyers GF, Luck R, Wildes CP, Tye KM (2016) Divergent routing of positive and negative information from the amygdala during memory retrieval. Neuron 90:348-361. CrossRef Medline

Beyeler A, Chang CJ, Silvestre M, Lévêque C, Namburi P, Wildes CP, Tye KM (2018) Organization of valence-encoding and projectiondefined neurons in the basolateral amygdala. Cell Rep 22:905918. CrossRef Medline

Burgos-Robles A, Kimchi EY, Izadmehr EM, Porzenheim MJ, Ramos-Guasp WA, Nieh EH, Felix-Ortiz AC, Namburi P, Leppla CA, Presbrey KN, Anandalingam KK, Pagan-Rivera PA, Anahtar M, Beyeler A, Tye KM (2017) Amygdala inputs to prefrontal cortex guide behavior amid conflicting cues of reward and punishment. Nat Neurosci 20:824-835. CrossRef

Corrado G, Doya K (2007) Understanding neural coding through the model-based analysis of decision making. J Neurosci 27:81788180. CrossRef

Ferenczi EA, Zalocusky KA, Liston C, Grosenick L, Warden MR, Amatya D, Katovich K, Mehta H, Patenaude B, Ramakrishnan C, Kalanithi P, Etkin A, Knutson B, Glover GH, Deisseroth K (2016) Prefrontal cortical regulation of brainwide circuit dynamics and reward-related behavior. Science 351:aac9698.

Hollerman JR, Schultz W (1998) Dopamine neurons report an error in the temporal prediction of reward during learning. Nat Neurosci 1:304-309. CrossRef

Knutson B, Samanez-Larkin G (2014) Individual differences in susceptibility to investment fraud, Stanford University. Accessed July 2018. Available at https://pdfs.semanticscholar.org/ 78a6/7f45aae9dd46c16fd10df668fddc39ed1bc1.pdf.

Knutson B, Samanez-Larkin GR, Kuhnen CM (2011) Gain and loss learning differentially contribute to life financial outcomes. PLoS One 6:e24390. CrossRef

Krabbe S, Gründemann J, Lüthi A (2017) Amygdala inhibitory circuits regulate associative fear conditioning. Biol Psychiatry 83:800-809.

Larkin JD, Jenni NL, Floresco SB (2016) Modulation of risk/ reward decision making by dopaminergic transmission within the basolateral amygdala. Psychopharmacology (Berl) 233:121-136. CrossRef

Namburi P, Beyeler A, Yorozu S, Calhoon GG, Halbert SA, Wichmann R, Holden SS, Mertens KL, Anahtar M, Felix-Ortiz AC, 
Wickersham IR, Gray JM, Tye KM (2015) A circuit mechanism for differentiating positive and negative associations. Nature 520:675678.

O'Neill P-K, Gore F, Salzman CD (2018) Basolateral amygdala circuitry in positive and negative valence. Curr Opin Neurobiol 49: 175-183. CrossRef Medline

Orsini CA, Trotta RT, Bizon JL, Setlow B (2015) Dissociable roles for the basolateral amygdala and orbitofrontal cortex in decisionmaking under risk of punishment. J Neurosci 35:1368-1379. CrossRef

Orsini CA, Hernandez CM, Singhal S, Kelly KB, Frazier CJ, Bizon JL, Setlow B (2017) Optogenetic inhibition reveals distinct roles for basolateral amygdala activity at discrete time points during risky decision making. J Neurosci 37:11537-11548. CrossRef

Piantadosi PT, Yeates DCM, Wilkins M, Floresco SB (2017) Contributions of basolateral amygdala and nucleus accumbens subregions to mediating motivational conflict during punished rewardseeking. Neurobiol Learn Mem 140:92-105. CrossRef

Preuschoff K, Mohr PNC, Hsu M (2015) Decision making under uncertainty. Lausanne: Frontiers Media SA.

Prévost C, McNamee D, Jessup RK, Bossaerts P, O’Doherty JP (2013) Evidence for model-based computations in the human amygdala during Pavlovian conditioning. PLoS Comput Biol 9:e1002918. CrossRef

Shabel SJ, Janak PH (2009) Substantial similarity in amygdala neuronal activity during conditioned appetitive and aversive emotional arousal. Proc Natl Acad Sci USA 106:15031-15036. CrossRef

St Onge JR, Stopper CM, Zahm DS, Floresco SB (2012) Separate prefrontal-subcortical circuits mediate different components of risk-based decision making. J Neurosci 32:2886-2899. CrossRef

Stopper CM, Tse MTL, Montes DR, Wiedman CR, Floresco SB (2014) Overriding phasic dopamine signals redirects action selection during risk/reward decision making. Neuron 84:177-189. CrossRef

Wächter T, Lungu OV, Liu T, Willingham DT, Ashe J (2009) Differential effect of reward and punishment on procedural learning. J Neurosci 29:436-443. CrossRef Medline

Weber EU, Johnson EJ (2009) Decisions under uncertainty. In: Neuroeconomics, pp 127-144. New York: Elsevier.

Zalocusky KA, Ramakrishnan C, Lerner TN, Davidson TJ, Knutson B, Deisseroth K (2016) Nucleus accumbens D2R cells signal prior outcomes and control risky decision-making. Nature 531:642-646. CrossRef 